\title{
Research Progress of Air Cycle Refrigeration Technology
}

\author{
XUE Xing ${ }^{1, a}$, WEI Fenglan ${ }^{1, b}$, LI Sheng ${ }^{1, c}$
}

\author{
${ }^{1}$ School of Architecture and Transportation Engineering, Guilin University of Electronic Technology, \\ Guilin, 541004, China \\ a276478298@qq.com; b2292387358@qq.com; '913820020@qq.com \\ Keywords: air cycle refrigeration, refrigeration principle, advantages, progress, application
}

\begin{abstract}
In response to the global call of environmental protection, a kind of environment-friendly air circulation refrigeration system is analyzed. Working principle of air cycle refrigeration system is introduced.The advantages of air refrigeration system are summarized, and main research achievements and progress in related fields at home and abroad are briefly narrated. The application in the field of the research of air cycle refrigeration are introduced as a clean and environment-friendly refrigeration way, in addition, the future development direction and the prospect of air cycle refrigeration technology are also predicted. Air cycle refrigeration technology will take up an important position in the future air conditioning field according the analysis.
\end{abstract}

\section{Introduction}

As a kind of traditional science and technology, refrigeration technology has penetrated into all fields of production, scientific research and daily life. Due to the limitation of the refrigerant of the commonly-used vapor compression refrigeration technology, scientists are developing alternative refrigerant actively. But the alternatives are not only expensive but also partly toxic. Although the destruction of the Ozone layer resulting from the use of CFCs is effectively avoided, the influence of the unknown risk on people's daily life cannot be ignored[1,2]. Air cycle refrigeration technology is a kind of environment-friendly and resource-saving mode of refrigeration, for using air as the refrigerant, the cost is low and dispose of air generating no pollution. In particular, air refrigeration technology has obvious advantages in small flow and cryogenic fields and it has been applied to train air conditioning, plane conditioning, food refrigeration and low temperature laboratory, almost all areas of refrigeration[3].

Many researchers have studied air cycle refrigeration in different levels.But most of them treated refrigerant air as dry air.However, air usually directly into air conditioning room in air conditioning system.Air temperature and pressure in the refrigeration process of system operation have great changes. At this moment air humidity will affect the performance of the system greatly, which may be accompanied by the condensation of water vapor in the air. Therefore the influence of air humidity on the refrigeration system can't be ignored[4].

\section{Working principle of air cycle refrigeration system}

From Figure 1 we can see that the air circulation system is mainly composed of compressor, expander, indoor and outdoor heat exchanger. Under the ideal condition, this refrigeration cycle includes two isobaric processes and two isentropic process. The circulating air gets into the compressor and becomes compressed, this is an isentropic process and the temperature and pressure rise(process 1-2); the compressed air of high temperature and high pressure gets into the outdoor heat exchanger and lets out heat isobaric, this is an isobaric process and the temperature falls(process 2-3); the air flow then gets into the expander and expands to the demanded temperature and pressure(process 3-4); the air flow of low temperature and low pressure gets into the indoor heat exchanger and absorbs the indoor heat, this is an isobaric process(process (process4-1), then the air flow gets into the compressor and the new refrigeration circulation begins. 


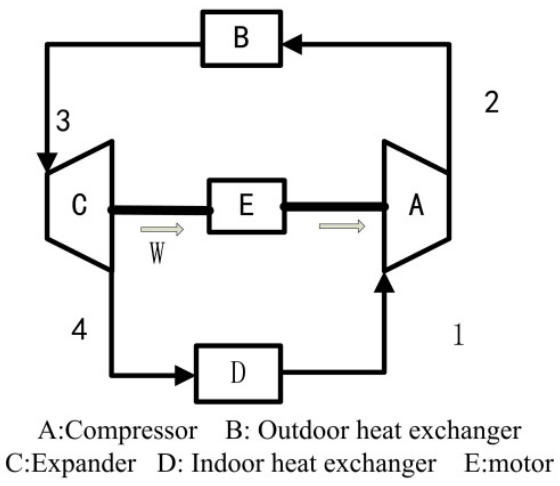

Fig. 1. Schematic diagram of the principle of air cycle refrigeration

\section{The advantages of air cycle refrigeration technology}

Air cycle refrigeration using air as the refrigerant, it thus has incomparable advantages over other refrigerants in the aspects of obtain, security and ecological environment demand and other aspects. (1) Friendly to environment and of no phase transformation, in this refrigeration cycle, the working air only plays the role of energy transfer, and its physical phase and chemical composition doesn't change, which is the most obvious characteristic of air refrigeration.

(2) The refrigeration temperature range is wide and the operating performance is excellent under low temperature. The refrigeration temperature can be adjusted in a wide range above $0{ }^{\circ} \mathrm{C}$, which can meet the requirement of working in the field of low temperature under $-30^{\circ} \mathrm{C}$ for a long time, and this is the unique advantage of air refrigeration system.

(3) Open refrigeration system can be adopted, the practical application process of air cooler is flexible, and so it can be arranged into an open system according to the actual situation, which is sending the low temperature air after expansion directly into the cold space to achieve refrigeration.

\section{Research progress of air cycle refrigeration}

Artificial refrigeration is realized by air refrigerating machine at early stage, however, the air cooling coefficient is low due to technical limit at that time. Compared with the appearance of steam refrigerator coming out subsequently, it has no advantage. Now, because of the appearance and development of turbine machinery, efficient and compact heat exchanger and air bearing, air refrigerator has been applied in the common field of air conditioning and refrigeration.

\subsection{Research progress in foreign countries}

An American John Ghauri made a closed cycle air cooler successfully in 1844. Due to technical limits, its coefficient of performance was low at that time, the application in industry is thus limited. Kanuua in Soviet Union used a turbine expander for the first time, and his experiment showed that the air flow of very low temperature can be obtained using an expander. In 1939, Kanuua used a turbine expander for air separation of supercritical refrigeration equipment, which provides the necessary technology and experience for the application of air refrigeration technology in aircraft. In 1991 Germany researched an air cooling machine applied to train air conditioner, and the installation and acceptance on ICE3 train was completed in 1998.

In 2001, the European Union began to study the application of air circulation system in building air conditioning system, their results show that the coefficient of refrigeration of an open air cycle refrigeration system is about 2 , the coefficient of heat generation is about 5 , higher than that of a traditional boiler system, and the total annual TEWI index of air circulation system is lower than that of conventional refrigeration system after comparison. S.W.T. Spence[5] researched an open 
double pressure boost air cooling device used in traffic and transportation. The results show that there exists an optimum pressure ratio in air refrigeration cycle, which makes the maximum refrigeration coefficient, and that the adding of an efficient reheater is beneficial to improve the coefficient of refrigeration, the refrigeration system after the optimization of components is working under full load. The energy consumption is $7 \%$ greater than that of a vapor compression refrigeration system. Elsayed S S researched the simulation and analysis of open air cycle refrigeration system under fixed and variable axial speed. Analysis results show that the cooling temperature of the system is decreased with the decrease of the cooling capacity under the condition of fixed axial velocity and the system can run under any refrigeration temperature when axial velocity is variable[6,7].

\subsection{Domestic research progress}

Researchers in China have conducted a lot of research work in the effects of improving the refrigeration efficiency and related parameters on the performance of refrigeration. Chen Chunzheng carried out theoretical investigation on air cycle refrigeration, the analysis and research of the establishment of thermal model, the influence factors of refrigeration coefficient and the temperature drop characteristic of the refrigerant are also researched. The results show that cycle pressure ratio, design parameter, component efficiency, air temperature and humidity and the inlet air temperature of compressor and expander all affect the refrigeration coefficient. Liu Yunxia[8-11] compared several feasible air refrigerator scheme used for the train in China, she points out that the most economic train air-conditioning refrigeration system is open negative pressure air refrigeration system, and that adopting reheating technology, spraying technology and multi-stage compression can improve the performance of the refrigeration system. In Zhang Zhenying's[12] research, the selection of pressure rate, the regulation method and greenhouse effect of the air refrigerator for train are analyzed, the results show that the pressure ratio of air refrigerating machine should be in the range of 2-2.5, and when adopting the regulation method of variable pressure rate, the coefficient of performance of this refrigeration system is 1-1.3, its TEWI value is less than that of the current steam compression refrigerator, and the discharge of greenhouse gas emission can be reduced.

\section{Application of air cycle refrigeration system}

Air cycle refrigeration technology has been applied to low temperature laboratory, train air conditioner, plane air conditioner, food refrigeration and mine cooling, further descriptions in every aspect are as follows:

\subsection{Application in low temperature laboratory}

As is shown in Figure 2, the air flow is cooled in the condenser after compressed in the compressor, the condensed water is separated in the water separator before entering the dry tower for further moisture remove, the air flow is compressed in centrifugal compressor after filtered, after passing through the water cooler and recooler, the temperature of the air reaches $0^{\circ} \mathrm{C}$ or less, then the air flow expands in the expander and cools the ambient air substantially. This low temperature air flow mixes with indoor air at certain proportion and then is sent indoors to absorb heat. 


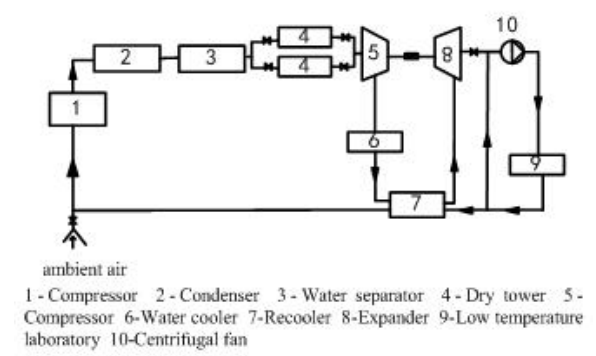

Fig. 2. Schematic diagram of air circulation system in low temperature environment testing room

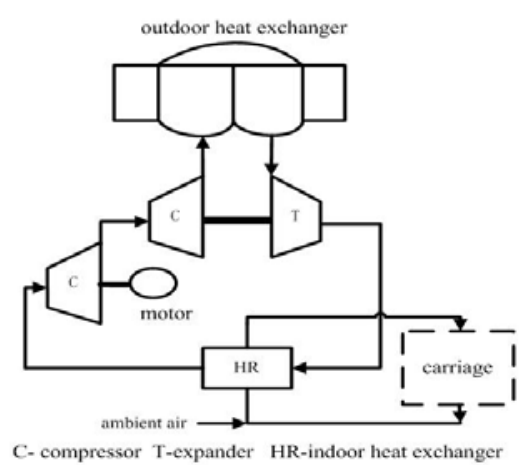

Fig. 3. Schematic diagram of air cooler for train air conditioning

\subsection{Application in air conditioner}

\subsubsection{Application in air conditioner for the train}

As is shown in Figure 3, the compressed air from the load heat exchanger enters the turbine expander for further pressure and temperature boost after the first compression, the discharged air of high pressure and temperature is cooled outdoors and then enters the expander, the air of low temperature and pressure is then used to cool the air in the carriage thus achieving the purpose of refrigeration.

\subsubsection{Application in plane air conditioner}

As is shown in Figure 4, air circulation refrigeration system is mostly used to regulate the cabin air in large aircraft, the compressed air from the engine is divided into the cold flow and the hot flow after precooled in the precooler, the cold flow enters the first stage heat exchanger for the first heat exchange then enters the compressor for pressure boost, after that the air flow is cooled in the secondary heat exchanger and the temperature and pressure falls in the expander. After mixing with the hot air flow from the temperature regulation valve, it is sent to the gas injection pump, driving the indoor cycle air and mixing with it at certain proportion,thus achieving the regulation of the indoor air.

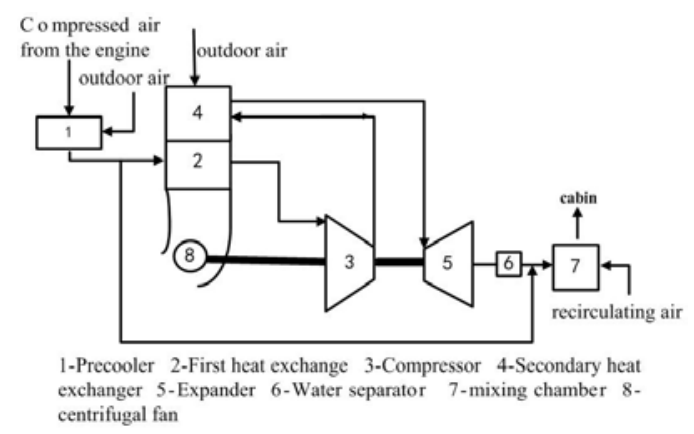

Fig. 4. Schematic diagram of the aircraft air conditioning cycle system 


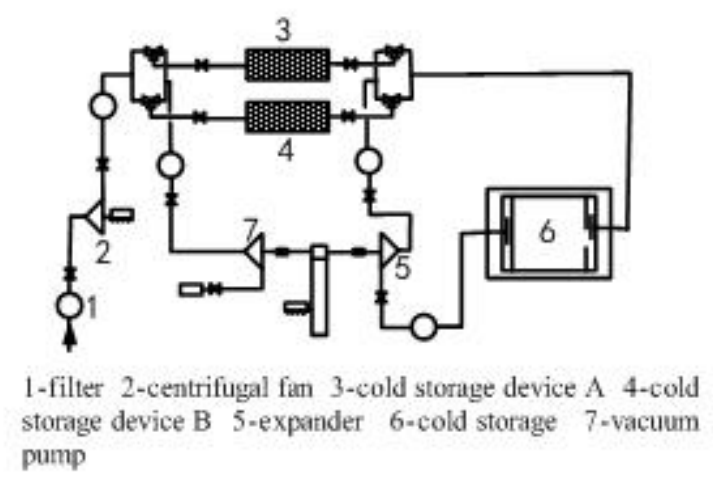

Fig. 5. Schematic diagram of air refrigeration in food freezing

\subsection{Application in food refrigeration}

As is shown in Figure 5, the air of the environment is inhaled via the centrifugal fan and filtered in the filter to remove impurities, the hot air of certain pressure enters the cold storage device A and exchange heat with the filler, the hot air is thus cooled then enters the cooling room, after absorbing heat, the air flow is cooled via the turbine cooler and then enters the cold storage device B to exchange heat with the filler, the cooled air is thus heated, then it is discharged into the atmosphere through a vacuum pump, a cycle is thus formed. In the freezing process, the contact of food with the cold surface and the refrigerant is not hoped, so the air refrigeration is the most appropriate method.

\section{Expectation}

Air refrigeration technology has made some progress in theory and practice, to meet the needs of production and life better, it is necessary to carry out relevant research work in the following aspects.

(1) When establishing the system model, the air leakage loss, the flow resistance of the heat exchanger and system pipeline should be taken into account, In the system modeling, the leakage loss, the system pipeline and the flow resistance of the heat exchanger should be taken into account, which will provide a more accurate and reliable theoretical basis for the actual production or experiment.

(2) The change of moisture content of wet air in expander should be researched in further step. The content of steam of wet air has a great influence on the refrigeration system. When the humidity is large, if the temperature of the air in the expander is lower than the dew point temperature, the vapor in the air will condense into droplets or even ice particle and release the latent heat, which makes the outlet temperature rise,thus reducing the available refrigerating capacity.

(3) The refrigeration efficiency needs to be improved. Because of the low specific heat and no phase change, air cycle refrigeration technology can only use the sensible heat, thus resulting in the small unit volume of cooling capacity, big volume flow and low coefficient of refrigeration.

\section{Summary}

Air is the optimum refrigerant which could meet the ecological requirements of the atmosphere, expect the good application in low temperature laboratory, plane air conditioner and food refrigeration, air cycle refrigeration system gets people attention gradually in other ordinary air conditioning refrigeration field. Though the refrigeration coefficient in this field limits its development in some extent, with the progress of industrial technology, the appearance of efficient 
refrigeration equipment and researchers' further research, air refrigeration technology will take up an important position in the future air conditioning field.

\section{Reference:}

[1] Zhao Chen.Experimental research in the high speed performance of air expander[D].Institute of Engineering Thermal Physics, Chinese Academy of Sciences(2013)

[2] Yu Hou,Shanju Yang,Xingya Chen,et al.Study on the matching performance of a low temperature reverse Brayton air refrigerator[J].Energy Conversion and Management ,2015(89):339 -348 .

[3] Huang Yanling,Chen Rudong.Application of air cycle refrigeration in cold storage vehicle[J].Refrigeration Air Conditioning and Electric Power Machinery,2013 (4): 32-34.

[4] Mohammad Hossein Ahmadi,Mohammad Ali Ahmadi.Thermodynamic analysis and optimization of an irreversible Ericsson cryogenic refrigerator cycle[J].Energy Conversion and Management,2015(89):147-155.

[5] S.W.T.Spence,W.J.Doran,D.W.Artt. Design,construction and testing of an air-cycle refrigeration system for road transport[J].International Journal of Refrigeration 2014,27(5):503-510.

[6] Elsayed S S, Miyazaki T, Hamamoto Y, et al. Performance analysis of air cycle refrigerator integrated desiccant system for cooling and dehumidifying warehouse[J]. International Journal of Refrigeration, 2012, 31(2): 189-196.

[7] Foster A M,Brown T,Gigiel A J,et al.Air cycle combined heating and cooling for the food industry[J].International Journal of Refrigeration,2011,34(5):1296-1304.

[8] Liu Yunxia,Zhao Yuanyang,Wang Shangjin.Scheme design of air cooling system for train air conditioner[J]. Fluid Machinery,2003, 31(6): 48-51.

[9] Guo Xiaohui,Guo Xianmin,Zhao Shuo.Numerical simulation of low temperature air cooling system[J].Cryogenics and Superconductivity,2015,43(5):65-70.

[10] Zhao Shuo. Simulation and experimental study on the steady state performance of low temperature air refrigeration system[D].Tian Jin:Tianjin University of Commerce,2013.

[11] Ling Rui. Simulation and experimental study on steady state performance of air cycle refrigeration system[D].Tian Jin:Tianjin University of Commerce,2014.

[12] Zhang Zhenying,Yang Yong,Chen Yanhua. Application analysis of air refrigerator in train air conditioner[J].Cryogenics and Superconductivity,2009,37(5):57-65. 